\title{
Development of a new segment to improve the dispersion of a nanofiller by extensional flow in a co-rotating twin-screw extruder
}

\author{
K. Matsumoto, Y. Arao \& T. Tanaka \\ Faculty of Science and Engineering, \\ Department of Mechanical Engineering, Doshisha University, Japan
}

\begin{abstract}
Extensional flow has been recognised as a more effective process than shear flow for improving the dispersion of nanofillers in polymeric materials. One of the production processes of nanocomposites is melt extrusion with a co-rotating twinscrew extruder (TSE), which is superior in terms of productivity and mixing performance. Then, we focused on "Blister Disk (BD)" having new segments which have many holes to generate the extensional flow in TSE. The purpose of this study is to optimize BD geometry (i.e. hole numbers, hole diameter and disk width) for improving the dispersion of nanocomposites. Primary to interpreting the mixing effect of $\mathrm{BD}$, the extensional flow state (i.e. extensional stress and pressure loss) at the hole entrance of BD was investigated with a 3D FEM analysis. Secondly, the mixing performance of the $\mathrm{BD}$ segment was evaluated by morphology observation (microscopy and TEM), rheological analysis and electric conductivity for polypropylene (PP) and carbon nanotube (CNT) nanocomposite. These experimental results were correlated with the results of the FEM analysis. The dispersion state of CNTs was improved with a decreasing hole area of BD and the disk width didn't affect the dispersion of CNT significantly. Also, it was found that the dispersion state of CNT can be controlled by a pressure drop at the $\mathrm{BD}$ segment.
\end{abstract}

Keywords: nanocomposite, extensional flow, twin-screw extruder.

\section{Introduction}

Recently, nanocomposites, which are reinforced by a nanofiller, have been developed to obtain excellent mechanical properties and functionalities. However, 
the expected properties of nanocomposites have not been obtained because of nanofiller agglomeration in the polymer. [1] Therefore, many solutions for dispersing the nanofiller have been developed. One of the promising nanocomposites production processes is melt extrusion with a co-rotating twinscrew extruder (TSE). TSE is superior in terms of high productivity and mixing performance. Thus, it is expected to disperse nanofillers by the TSE process.

Usually, shear flow mixing is widely used to disperse the nanofiller agglomerations in the melting polymer. However, Grace [2] investigated that shear flow mixing is energetically inefficient, and it is limited to a low value of viscosity ratio $(<4)$ for polymer blending (as shown in fig. 1) [3]. Extensional flow mixing has several advantages compared with shear flow mixing, namely, there is 1) no viscosity ratio limit and 2) better dispersive and distributive mixing. Then, we attempted to create extensional flow in TSE with a "blister disk (BD)" which is original segments of Coperion [3] (as shown in fig. 2) for dispersing the nanofillers.

To disperse the CNT agglomerates, the stress magnitude is an important parameter (Manas et al. [4]). In our previous study, it was revealed that higher extensional stress can occur than with other conventional mixing segments such as kneading segment by FEM analysis. Furthermore, a better dispersion state of CNT was obtained with a BD segment experimentally. The compounding by extensional flow might be an excellent method for producing well dispersed nanocomposites.

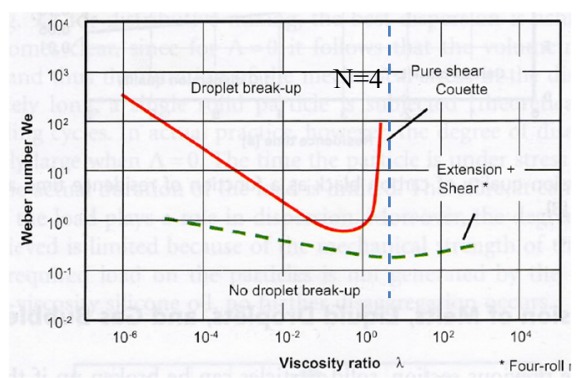

Figure 1: Grace curve (extensional flow is more effective than shear flow for mixing performance).
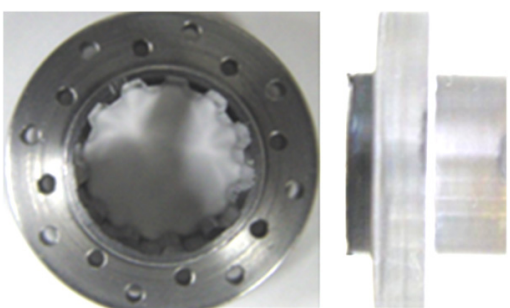

Figure 2: Geometry of blister disk (it has many small holes).

However, the geometry of BD was not optimized. The objective of this study is the optimization of BD geometry by changing hole numbers, hole diameter and disk width to improve the dispersion state of nanofillers. To interpret the mixing effects of a BD segment, it is important to investigate the shear and extensional stress magnitude by FEM analysis. After that we conducted the experiments to reveal which parameters (BD geometry) affect the dispersion state of nanofiller, and correlated with analytical results. Moreover, the shear and extensional stress can be calculated from pressure drop by Hagen Poiseuille and Cogswell [5]. In addition, the pressure drop can be monitored experimentally by pressure 
transducer. Therefore, the pressure drop can be the most important parameter to evaluate the performance of a BD segment. Thus, the relationship between pressure drop and dispersion state of CNTs was investigated in this study.

\section{3D FEM numerical simulation}

The extensional flow state was analysed by using the commercial ANSYS POLYFLOW 14.5 code. The following assumptions were used as flow conditions: I) fluid is incompressible and viscous non-Newtonian; II) the flow channel is fully filled with melting polymer; III) inertia and gravitational force are negligible; IV) the fluid sticks on the barrel surface; and V) the flow is isothermal. From these assumptions, these equations of mass, momentum and constitutive equation with assumptions are given as follows:

$$
\begin{gathered}
\nabla \cdot \mathbf{v}=0 \\
-\nabla P+\nabla \boldsymbol{\tau}=0 \\
\boldsymbol{\tau}=2 \eta \mathbf{D}
\end{gathered}
$$

where $\mathbf{v}$ is velocity vector, $P$ is hydrostatic pressure, $\boldsymbol{\tau}$ is extra stress tensor, and $\mathbf{D}$ is rate of deformation tensor. The material used in this study was PP and multiwall carbon nanotube (MWCNT). Detail of the materials are described at the experimental section. The viscosity of PP/CNT (1wt\%) nanocomposites was measured by rotational rheometer (Bohlin Gemini II) and capillary rheometer (Rosand RH2200). The viscosity curve and curve fitted results are shown in fig. 3. Extensional viscosity was measured by entrance pressure drop with Cogswell's method [5]. The non-Newtonian viscosity for shear and elongation were described by the Carreau-Yasuda model and Sarkar-Gupta model [6] as follows:

$$
\begin{gathered}
\eta_{s}=\eta_{0}\left[1+(\lambda \dot{\gamma})^{a}\right]^{\frac{n-1}{a}} \\
\eta_{e}=\eta_{0}\left[3+\delta\left\{1-\frac{1}{\sqrt{1+\left(\lambda_{1} \dot{\varepsilon}\right)^{2}}}\right\}\right]\left[1+\left(\lambda_{2} \dot{\varepsilon}\right)^{2}\right]^{\frac{m-1}{2}}
\end{gathered}
$$

where $\eta_{s}$ and $\eta_{e}$ are simple shear and uniaxial extensional viscosity. The model parameters are shown in table 1. The shear rate and stretch rate [7] can be expressed by the following equations:

$$
\begin{aligned}
& \dot{\gamma}=\sqrt{2 I I_{D}} \\
& \dot{\varepsilon}=6 I I I_{D} / I I_{D}
\end{aligned}
$$

where, $I I_{D}$ and $I I I_{D}$ are the second invariant and third invariant of the rate of deformation tensor. In the case of using these equations for converging flow, the shear and extensional state can be separated (as shown in fig. 4). The stress 
magnitude was introduced to evaluate the dispersion state of PP/CNT nanocomposites and it can be calculated from strain rate and viscosity. The analysis model was shown in fig. 5. Then, shear and extensional stress magnitude for each BD segment was calculated by changing the geometry (as shown in table 2). As boundary conditions, the barrel temperature was $200^{\circ} \mathrm{C}$, and the flow rate was $4.5 \mathrm{~kg} / \mathrm{h}$. The flow rate at the inlet cross section was constant and the pressure at the outlet cross section was set to be zero. There is no slip on barrel surface.

Table 1: $\quad$ Parameter for fitting models.

\begin{tabular}{|c|c|c|c|c|c|c|c|}
\hline & $\eta_{0}$ & $\lambda$ & $\lambda_{1}$ & $\lambda_{2}$ & $n$ & $m$ & $\delta$ \\
\hline Carreau-Yasuda Model & 1080 & 0.006 & & & 0.1 & & \\
\hline Sarkar-Guputa Model & 1080 & & 0.5 & 0.2 & & 0.5 & 3 \\
\hline
\end{tabular}

Table 2: Geometry condition of BD for 3D FEM numerical simulation.

\begin{tabular}{|c|c|c|c|}
\hline & $\begin{array}{c}\text { (a) Difference } \\
\text { of hole } \\
\text { diameter }\end{array}$ & $\begin{array}{c}\text { (b) Difference } \\
\text { of hole } \\
\text { number }\end{array}$ & $\begin{array}{c}\text { (c) Difference } \\
\text { of disk width }\end{array}$ \\
\hline Hole diameter $\mathrm{D}[\mathrm{mm}]$ & $\mathrm{D}=0.5 \sim 1.2$ & $\mathrm{D}=1.0$ & $\mathrm{D}=1.0$ \\
\hline Hole number $\mathrm{N}[-]$ & $\mathrm{N}=16$ & $\mathrm{~N}=8 \sim 32$ & $\mathrm{~N}=16$ \\
\hline Disk width $[\mathrm{mm}]$ & $\mathrm{W}=3.0$ & $\mathrm{~W}=3.0$ & $\mathrm{~W}=1.5 \sim 6.0$ \\
\hline
\end{tabular}

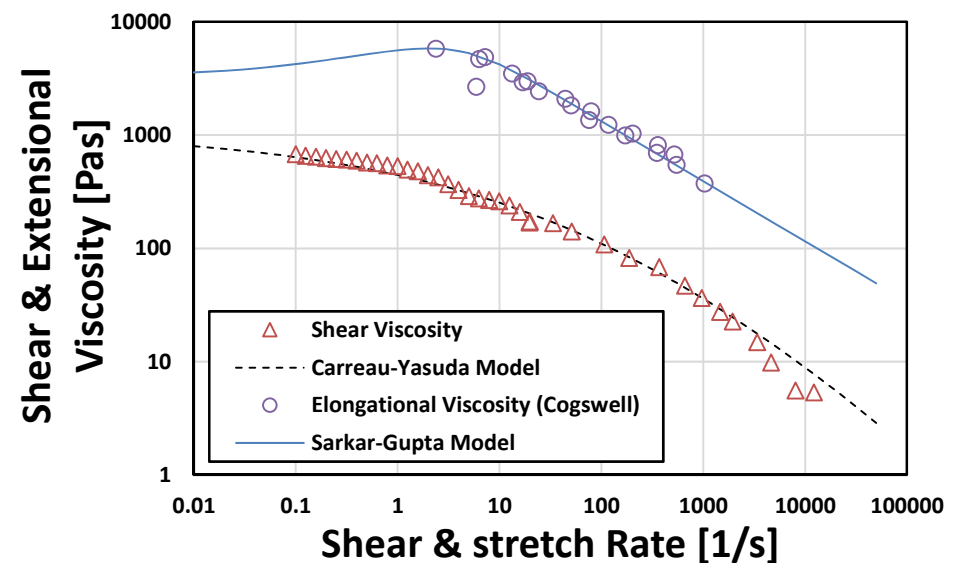

Figure 3: Viscosity data of PP/CNT nanocomposite and curve fitted results by the Carreau-Yasuda model and Sarkar-Gupta model. 


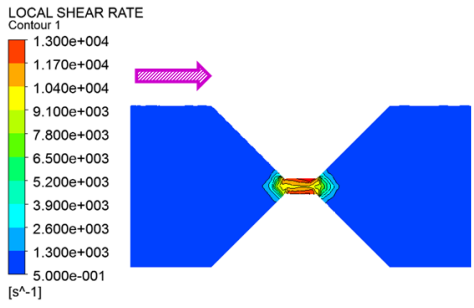

(a)

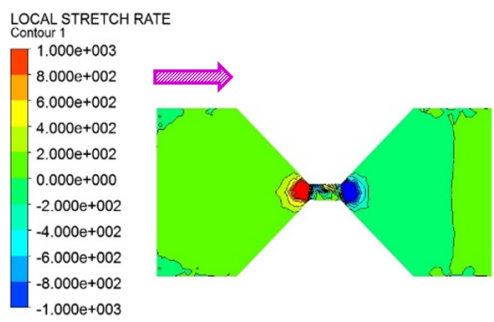

(b)

Figure 4: Flow state of converged flow: (a) shear flow and (b) extensional flow.

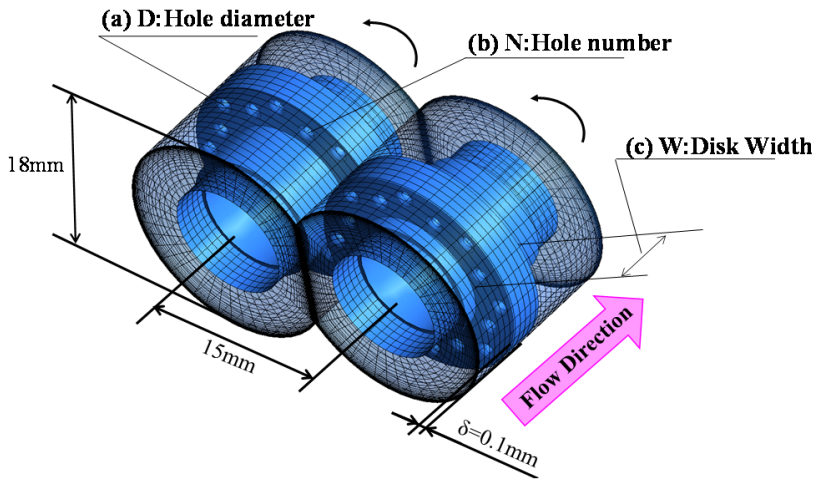

Figure 5: FEM model of blister disk (nodes: 158900, elements: 149872).

\section{Experimental}

\subsection{Materials and preparation of PP/CNT nanocomposite sheet}

In this study, PP/CNT nanocomposite was used as the model of nanocomposite to evaluate the mixing performance of various BD segments. PP J108M with MFI 45g/10min (Prime Polymer Co., Ltd.) was used as matrix. MWCNT (NC 7000 Nanocyl) was used as nanofiller. The average length and average diameter of the CNT are $1.5 \mu \mathrm{m}$ and $9.5 \mathrm{~nm}$, respectively.

These materials were compounded with co-rotating TSE (ZSK18 MegaLab, Coperion $\mathrm{GmbH}$ ) at $200^{\circ} \mathrm{C}$ and $150 \mathrm{rpm}$. As the method of compounding process, the master batch process was used to get uniformly compounded pellet. Firstly, PP and CNT were blended with dry condition at RT with 5.0wt\% CNT. Then, the dryblend materials were compounded by twin-screw extruder and obtained the $5.0 \mathrm{wt} \%$ of $\mathrm{PP} / \mathrm{CNT}$ pellets. Then, the obtained $\mathrm{PP} / \mathrm{CNT} 5.0 \mathrm{wt} \%$ pellets were diluted to $1.0 \mathrm{wt} \%$ by adding PP pellets using the same BD segments.

The screw configuration is shown in fig. 6. The TSE screw consisted of kneading segments for plasticization of PP pellets, full flight segment for 
conveying a screw with a little mixing effect, and the various geometries of BD segments. The BD geometries were changed as follows (in fig. 7): (a) different hole diameter $(\mathrm{D}=0.5 \mathrm{~mm}, 1.0 \mathrm{~mm}, 1.2 \mathrm{~mm})$, (b) different hole numbers $(\mathrm{N}=8,16$, $32)$, and (c) different disk lengths $(\mathrm{W}=1.5 \mathrm{~mm}, 3.0 \mathrm{~mm}, 4.5 \mathrm{~mm})$. The extruded $\mathrm{PP} / \mathrm{CNT}$ nanocomposites were collected directly, and the collected samples were formed to $2 \mathrm{~mm}$ thickness sheet by compression moulding. Compression moulded samples were used for the observation samples.

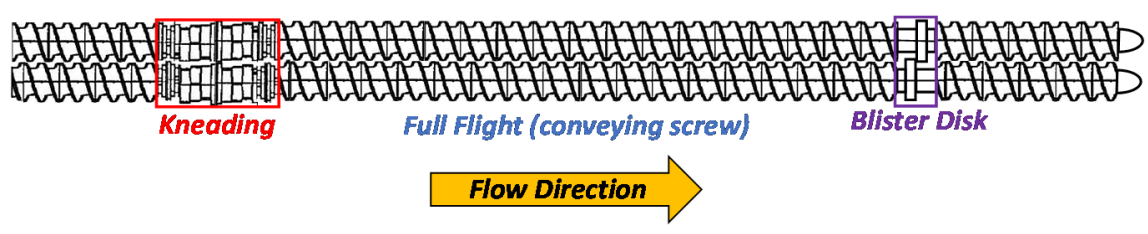

Figure 6: Screw configuration of a twin screw extruder.

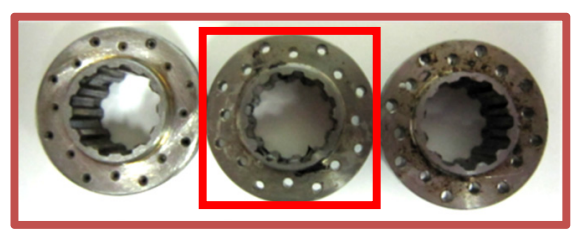

(a)

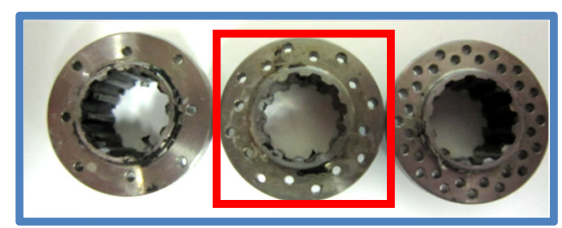

(b)

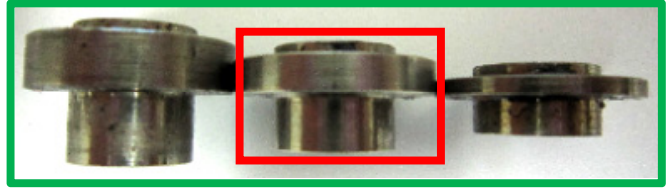

(c)

Figure 7: $\quad$ BD geometries difference of (a) hole diameter (D0.5, D1.0, D1.2), (b) hole numbers (N8, N16, N32) and (c) disk width (W4.5, W3.0, W1.5). Red lines indicate the same condition (i.e. standard).

\subsection{Morphological characterization}

The morphology of nanocomposite was studied through optical microscopy and transmission electron microscopy (TEM) using MEIJI TECNO MT9430 and JEOL JEM-2100F, respectively. The obtained PP/CNT sheets were cut to $2 \mu \mathrm{m}$ and $100 \mathrm{~nm}$ specimens for observing with microscopy and TEM, respectively.

In the case of microscopy observation, the 9 specimens were cut from variety place of one PP/CNT sheet for each geometry condition of BD segments and observed with $230 \times$ magnifications. If the thickness is thin enough to transmit the light of optical microscope, the CNT agglomerates can be clearly detected. The area of CNT agglomerates decreases with improving CNT dispersion, due to the resolution of the optical microscope. To evaluate quantitatively, observed 
images were analysed with sigma scan pro 5 and the agglomeration area of CNTs were calculated and averaged. In the case of TEM observation, an acceleration voltage of $200 \mathrm{kV}$ was employed.

\subsection{Rheological measurement}

The rheological measurements were carried out by rotational rheometer (Gemini II, Bohlin Instruments) equipped with $25 \mathrm{~mm}$ cone plate geometry with a cone angle of $5.4^{\circ}$. Oscillation measurements were performed for each specimen at $200^{\circ} \mathrm{C}$ and frequency $\omega$ range from 0.018 to $100 \mathrm{rad} / \mathrm{s}$ with $1 \%$ strain. Storage modulus $\mathrm{G}^{\prime}$ and complex viscosity $\eta^{*}$ were measured and these parameters increase as the dispersion state of CNT increases due to the increase of interfacial interaction of PP and CNTs $[8,9]$. The complex viscosity $\eta^{*}$ was able to fit with Carreau-Yasuda with yield stress. This equation is given as follows:

$$
\eta^{*}=\frac{\sigma_{0}}{\omega}+\eta_{0}\left[1+(\lambda \omega)^{a}\right]^{(n-1) / a}
$$

where $\sigma_{0}$ is yield stress, $\omega$ is frequency, $\eta_{0}$ is zero shear viscosity, and $\lambda, n, a$ are material parameters. From this equation, the yield stress of starting flow can be calculated and used as quantitative evaluation parameter for CNT dispersibility.

\subsection{Electrical measurement}

Electrical properties were measured with Loresta GP (Mitsubishi Chemical Analytech Co., Ltd.) for three specimens according to JIS K 7194. The specimen size was $50 \times 80 \times 2 \mathrm{~mm}$. The average volume resistivity was calculated. As improving the dispersion state of CNT, the $3 \mathrm{D}$ network structure are constructed and the volume resistivity are decreased. Therefore, volume resistivity can be used as a quantitative evaluation parameter for CNT dispersibility.

\section{Results and discussion}

\subsection{The results of 3D FEM numerical simulation}

The effects of hole diameter, hole numbers and disk width for the shear and extensional stress were investigated by FEM analysis. Fig. 8 shows the maximum of shear stress and extensional stress results. With respect to the effect of hole diameter and hole number, the hole area (i.e. total area of BD hole) was adapted as the horizontal axis. According to the analytical results, the maximum of shear and extensional stress were increased with decreasing the hole area. Therefore, smaller hole area (i.e. smaller hole diameter and hole numbers) is expected to disperse CNT agglomerates effectively. However, in the case of a hole diameter of $0.5 \mathrm{~mm}$, the shear and extensional stress are decreased dramatically. The reason can be considered because of linkage at the gap of the BD disk. 
On the other hand, regarding the disk width, shear and extensional stress were decreased slightly as the disk width became larger. However, significant changes were not observed compared with the difference of hole diameter and numbers. Therefore, it is considered that the disk width does not affect the dispersion of CNT significantly.

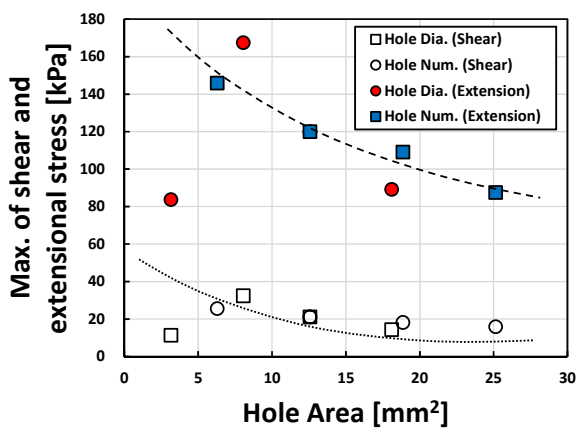

(a)

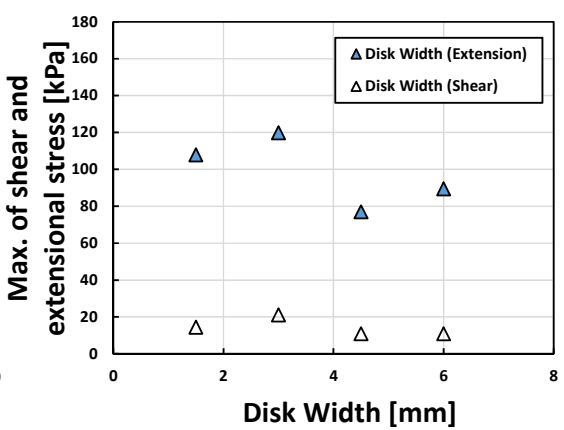

(b)

Figure 8: Shear and extensional stress data of 3D FEM for the (a) difference of hole diameter and numbers and (b) difference of disk width.

\subsection{Experimental results}

\subsubsection{Morphological characterization}

Fig. 9 shows the observation images of optical microscopy. To discuss these results more quantitatively, the average agglomeration area was analysed and is shown in fig. 10. According to these results, the agglomeration area decreased as the hole area was smaller (smaller diameter and hole numbers). On the other hand, in the case of disk width, the agglomeration area was slightly decreased with decreasing disk width. These results are well explained by the analytical results shown in fig. 8. Shear and extensional stress increase as the hole area decreases. Hence, the reduction of hole area leads to a dispersion of CNT.

Moreover, TEM observation results of the smallest hole area (D0.5) and largest hole area (N32) are shown in fig. 11. In the case of the results of the smallest hole area, many dispersed CNTs were observed. On the other hand, lots of CNT agglomerates were observed for the $\mathrm{BD}$ with the largest hole area.

\subsubsection{Rheological characterization}

The results of storage modulus G' and complex viscosity $\eta^{*}$ were shown in fig. 12 . According to these results, storage modulus and complex viscosity were increased with improving the dispersion state of CNT at lower frequency (e.g. $\omega=0.018 \mathrm{rad} / \mathrm{s}$ ). Then, the fitted curves with Carreau-Yasuda equation for complex viscosity are well fitted (as shown in fig. 12). Each parameter was $a=3$, $n=0.8, \lambda=80, \eta_{0}=1000$, respectively. From this fitting curve, the yield stress $\sigma_{0}$ can be calculated and these rheological characteristics can be evaluated quantitatively with yield stress. Fig. 13(a) shows the yield stress vs. the hole area. According to 
these results, the tendency of increasing the yield stress as a smaller hole area can be seen in the case of the difference of hole diameter and numbers. This tendency is corresponded to the tendency of morphological results.

On the other hand, regarding the effect of disk width, the above tendency was not observed. This is because the shear and extensional stress do not change significantly with the difference of disk width (from fig. 8).

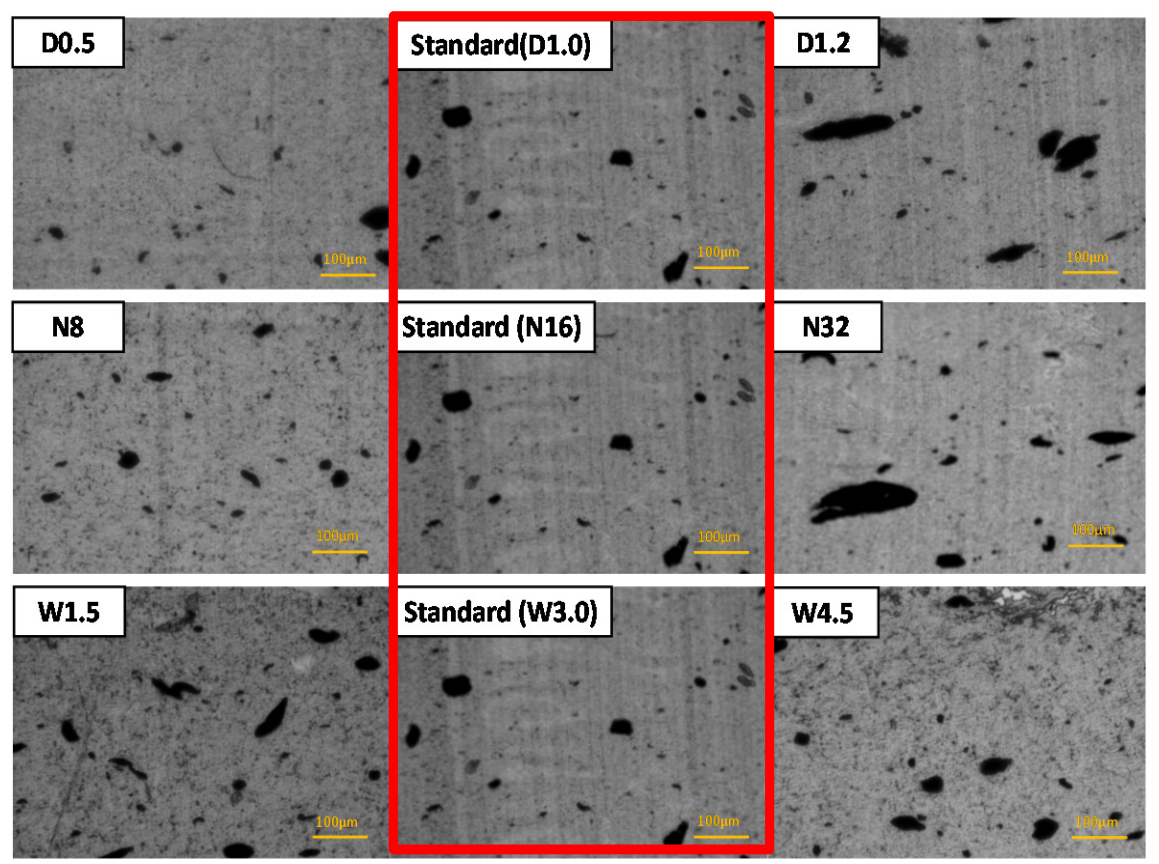

Figure 9: Optical microscopy images (the red line indicates the same BD geometry).

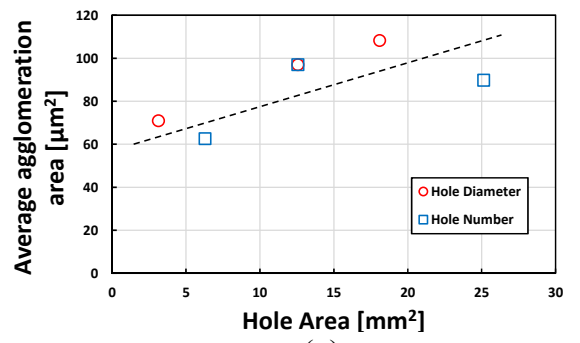

(a)

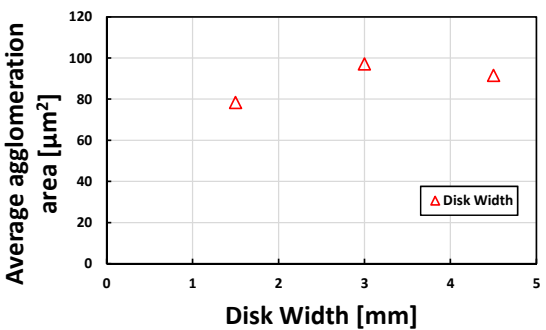

(b)

Figure 10: Average agglomeration area of CNT data with image analysis for the difference of hole diameter, hole numbers and (b) disk width. 


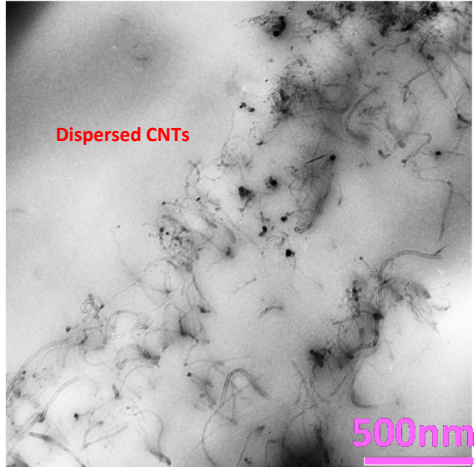

(a)

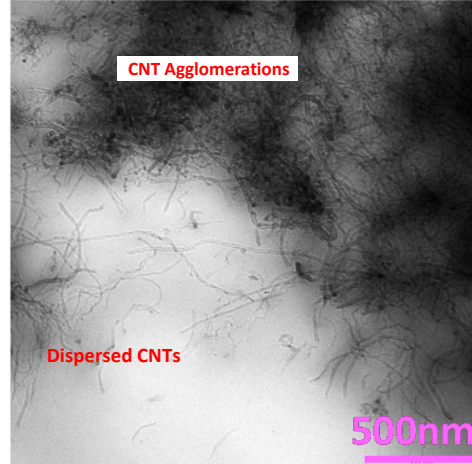

(b)

Figure 11: TEM observation images of (a) smallest hole area (D0.5) and (b) largest hole area (N32).

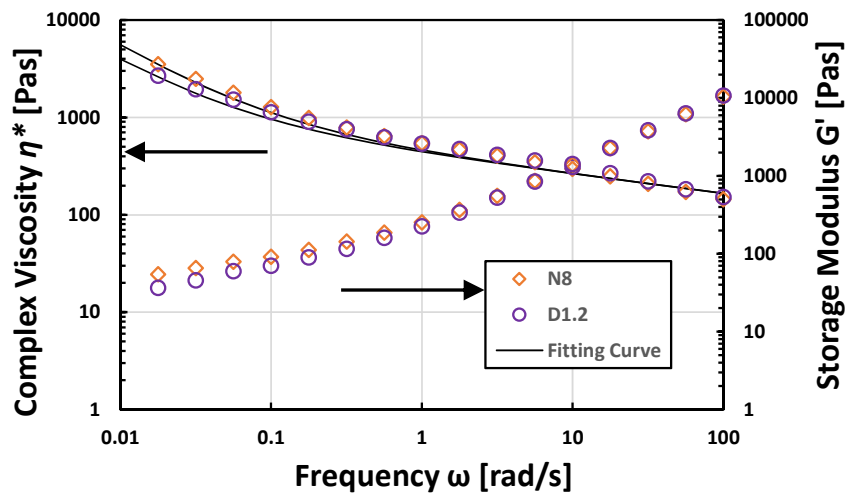

Figure 12: Variation of the storage modulus $\mathrm{G}^{\prime}$ and complex viscosity $\eta^{*}$, and fitted results with Carreau-Yasuda equation for viscosity.

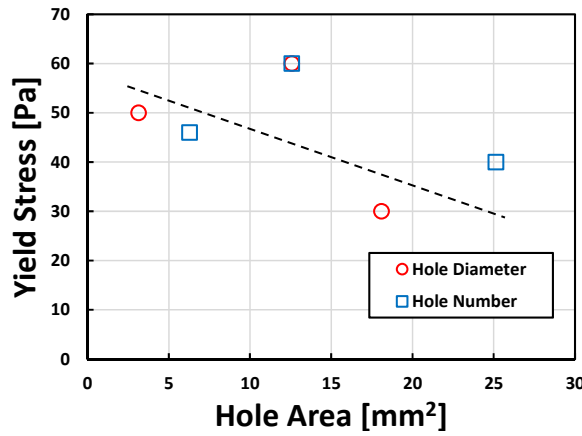

(a)

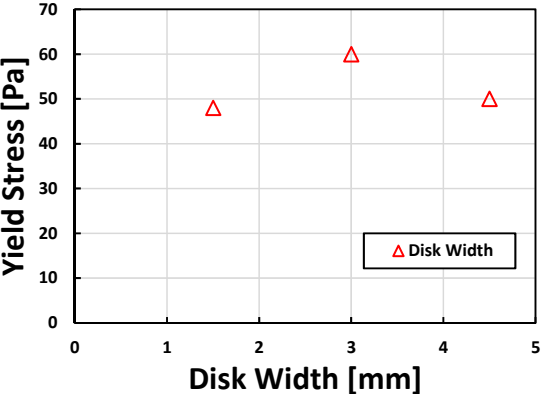

(b)

Figure 13: Calculated yield stress data for (a) difference of hole diameter, hole numbers and (b) disk width. 


\subsubsection{Electrical characterization}

In the same way, the volume resistivity results are shown in fig. 14. According to these results, in the case of the difference of hole diameter and numbers, the volume resistivity was decreased as smaller hole area. This tendency matches the result of morphological and rheological measurements. Therefore, decreasing the hole area was found to improve the dispersion state of CNT and the electrical conductivity. On the hand, with the difference of disk width, volume resistivity was decreased slightly as smaller disk width. This result also corresponds to the simulation results.

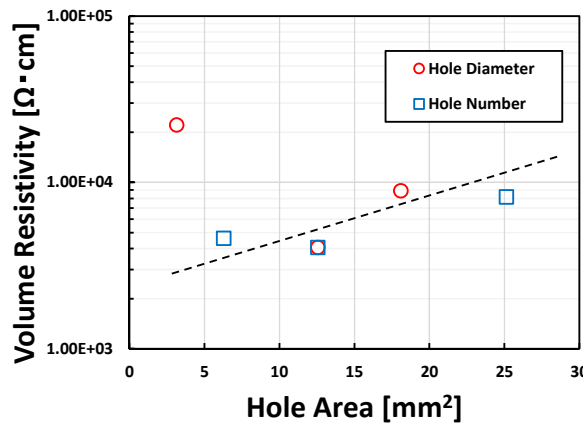

(a)

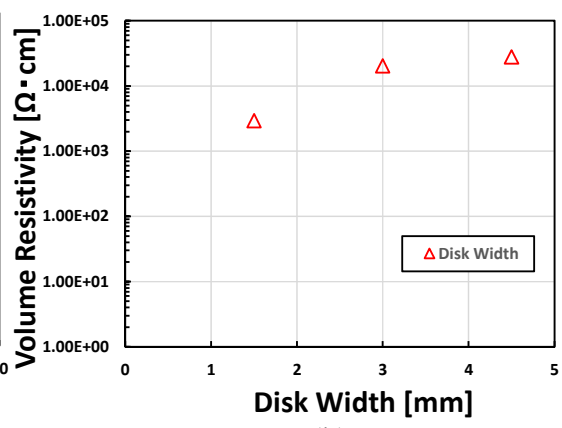

(b)

Figure 14: Volume resistivity data for (a) difference of hole diameter, hole numbers and (b) disk width.

\subsection{The relationship between pressure drop and CNT dispersibility}

According to these results, CNT dispersion can be dominated by shear and extensional stress. However, these important parameters, i.e. shear and extensional stress, cannot be monitored directly in the TSE process. Therefore, the pressure drop, which can be monitored in the process, was focused and stress magnitude can be calculated from the pressure drop. The shear stress can be calculated from the Hagen-Poiseuille equation and extensional stress can be calculated from the Cogswell equation. The pressure can be divided as follows:

$$
\Delta P_{\text {total }}=\Delta P_{\text {entry }}+\Delta P_{\text {shear }}+\Delta P_{\text {out }}
$$

where $\Delta P_{\text {total }}$ is total pressure drop, $\Delta P_{\text {entry }}$ is entrance pressure drop, $\Delta P_{\text {out }}$ is exit pressure drop, and $\Delta P_{\text {shear }}$ is shear pressure drop.

The relationship of each stress magnitude and pressure drops were investigated with 3D numerical simulation. Fig. 15 shows the relationship of each pressure drop and maximum stress magnitude. About the shear effect, as increasing the shear pressure drop, the maximum shear stress increased like exponentially. On the other hand, with respect to the extension effect, the extensional stress increased lineally by the sum of entrance and exit pressure drop. The correlation between pressure drop and stress magnitude was confirmed, and the dispersion state of CNT with 
respect to $\mathrm{BD}$ segments can be estimated by the pressure drop. Hence, it is considered that in the case of the screw composed of BD segments, the dispersion of CNT can be controlled with on-line system using a pressure transducer.

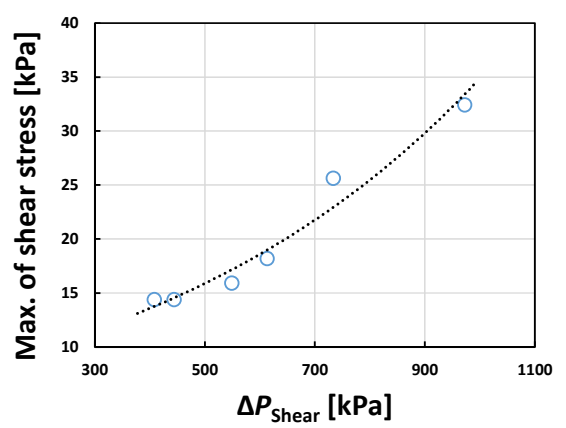

(a)

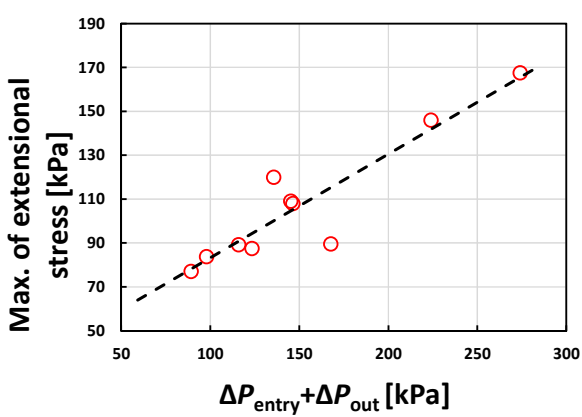

(b)

Figure 15: The relationship pressure drop and stress magnitude. (a) Shear pressure drop vs. max. shear stress, and (b) entrance and exit pressure drop vs. max. extensional stress.

\section{Conclusion}

In this study, the mixing performance of new segments for improving the dispersion state of a nanofiller by extensional flow was investigated by numerical simulation and a series of experiments. Shear and extensional stress for each BD segment were quantified by numerical simulation, and these results back up the understanding of the experimental results. According to these results, the CNT dispersion increased as the hole area became smaller (i.e. smaller hole diameter and numbers) of $\mathrm{BD}$, because the shear and extensional stress increases with decreasing hole area. On the other hand, regarding the effect of disk width, the CNT did not change significantly because the disk width slightly affects the stress in the flow. Then, the relativity of pressure drop and stress magnitude were confirmed by numerical simulation. The improving of CNT dispersion can be caused by giving a higher pressure drop (i.e. higher stress magnitude) and can be controlled with an on-line system by setting the pressure transducers to TSE.

\section{Acknowledgement}

The authors acknowledge support from Doshisha University Research and Development Center for Advanced Composite Materials.

\section{References}

[1] Ma, P.C., Siddiqui, N.A., Marom, G., Kim, J.K., Dispersion and functionalization of carbon nanotubes for polymer-based nanocomposites: A review, Composites: Part A, vol. 41, pp. 1345-1367, 2010. 
[2] Grace, H.P., Dispersion Phenomena in High Viscosity Immiscible Fluid Systems And Application of Static Mixers As Dispersion Devices in Such Systems, Chem. Eng. Comm., Vol. 14, pp. 225-277, 1982.

[3] Kohlgruber, K., Co-Rotating Twin-Screw Extruders, Carl Hanser Verlag GmbH \& Co. KG, p. 170, 2007.

[4] Manas, Zlogzower, I., Nir, A. and Tadmor, Z., Dispersive Mixing in Internal Mixers - A Theoretical Model Based on Agglomerate Rupture, Rubber Chemical Technol., Vol. 55, pp. 1250-1285, 1982.

[5] Cogswell, F.N., Converging flow of polymer melts in extrusion dies, Polym. Eng. Sci. Vol. 12, pp. 64-73, 1972.

[6] Sarkar, D. and Gupta, M., Further Investigation of the Effect of Elongational Viscosity on Entrance Flow, J. Reinf. Plast. Comp., Vol. 20, pp. 1473-1484 2003.

[7] Debbaut, B. and Crochet, M.J., Extensional effects in complex flows, J NonNewtonian Fluid Mech. Vol.30, pp.169-184, 1988.

[8] Lertwimolnun, W., Vergnes, B., Influence of compatibilizer and processing conditions on the dispersion of nanoclay in a polypropylene matrix, Polymer, Vol. 46, No. 10, pp. 3462-3471, 2005.

[9] Lertwimolnun, W., Vergnes, B., Effect of Processing Conditions on the Formation of Polypropylene/Organoclay Nanocomposites in a Twin Screw Extruder, Polymer Engineering and Science, Vol. 46, No. 3, pp. 314-323, 2006. 